\title{
Simple method of selective harmonic tracking (SHT) of signals in an integrated AC-DC power system
}

\author{
K.G. Narendra \\ H.S. Chandrasekharaiah
}

Indexing terms: Selective harmonic tracking, Fourier synthesis. Multiple pulse converters

Abstract: The objective of the paper is to present a simple method of finding the harmonic coefficient of a trigonometric Fourier series representation for signals which are defined over a finite range. This method can be used selectively to track any harmonic component or range of harmonic components of the signal. A matrix method has been developed which uses the least square (LSQ) criterion to approximate the given signal with that of the assumed Fourier series representation. The method can be used, in general, as signal processing tool. For the purpose of illustration, a digital computer program is developed for selective harmonic tracking (SHT) of waveforms generated in multiple pulse converter systems connected to an HV AC system.

\section{Introduction}

It is well known that the operation of an AC-DC system consisting of converters, which is basically a switching device, generates harmonics whose detrimental effects such as overheating, resonance overvoltages, communication interference, false tripping of relays etc., must be limited for the reliable operation of the system with minimum interruption. It is usual practice to install a range of filters for this purpose. In designing these filters, individual harmonic distortion (IHD) or total harmonic distortion (THD) expressed with reference to the fundamental frequency component is an important index. Therefore, evaluation of the fundamental frequency component which has a major contribution in the distorted signal is of greater importance.

Many transformation techniques, such as DFT, FFT, Hartley transform etc., are available for calculating the Fourier components which have their own advantages and disadvantages [1-3]. For the estimation of harmonics in power systems, the Kalman filtering approach [4, 5] and a state estimation methodology using a power series expansion [6] are employed. Their identification is described in the recent literature based on a revised group method of data handling [7] and using neural net- works [8]. In these methods the algorithms tend to be complex and the computation time is generally high. The DFT method cannot handle signals with partial disturbances; nor can these methods be applied to nonuniformly sampled signals.

The advantage of the infinite variety of ways to establish Fourier trigonometric series representation for a defined finite region [9] function is used here. From an engineering point of view, i.e. for practical reasons including the reduction of computational labour and overcoming the disadvantages mentioned above, the present method of finding the selective Fourier component is simple and effective. By selective, we mean any rth component of the sine and cosine term in the trigonometric series:

$$
y(t)=a,+\sum_{r=1}(a, \sin r \omega t+\boldsymbol{b}, \cos r \omega t)
$$

This can be tracked using a method which is described in the following Section. Hence the required component of the signal may be easily extracted from an electrically polluted wave form. This kind of filtering is very helpful in assessing the performance of the AC-DC power system, especially in a high impedance system (i.e. weak $\mathrm{AC}$ system) which is predominantly inductive, the filter and compensating capacitors leads to a possibility of lower order resonance which affects the reliability of the system performance. In flexible alternating current transmission (FACT) the use of modern reactive power compensators, such as thyristor controlled reactors (TCRs) and thyristor switched capacitors (TCRs) which further injects harmonics at the point of common coupling, can cause harmonic instability and create resonance overvoltages at lower order harmonic frequencies. In digital relaying, the number of harmonics needed is in the order of 4-20. The fundamental frequency component alone is used in impedance relaying, while a few harmonics (fundamental, second and fifth) are used in transformer algorithms [10]. The present method is also suitable for these applications.

If the contribution of the fundamental is evaluated, a criterion can be set as to whether or not to calculate the other harmonic components. For example, if THD of $\mathbf{3} \%$ is allowed in a system, then this can be verified qualitatively by tracking the fundamental frequency component alone or a particular harmonic or a range of harmonic components contained in the signal using the present SHT method. The frequency components calculated assumes LSQ criterion which is discussed in the following section. 
We know, by the Fourier trigonometric series expansion, that a given function can be expressed by the linear combination of the sine and cosine terms, provided that the function satisfies Dirichlet conditions, as

$$
\begin{aligned}
s(t) \simeq & y(t) \\
= & a_{0}+\mathrm{a}, \sin o t+b, \cos \omega t \\
& +\mathrm{a}, \sin 2 \omega t+b, \cos 2 \omega t \mathbf{t} \\
& +\mathrm{a}, \sin m o t+b_{m} \cos m o t
\end{aligned}
$$

where $s(t)$ is approximate function of $y(t)$. Over the fundamental range and for $p$ values (samples) of $y(t)$, eqn. 1 can be expressed in matrix form as

$$
\boldsymbol{y}=A \boldsymbol{x}
$$

where $y_{p \times}$, is a column vector, $A,$, , is a matrix of sine and cosine terms, where $p>m$ (over determined system) and $\mathbf{x}_{x}$, is a column vector consisting of the harmonic components to be tracked.

To have the best approximation of $\boldsymbol{A x}=\boldsymbol{y}$, let the residual error

$$
\varepsilon=(A x-y)
$$

Using the LSQ criterion,

$$
\varepsilon^{2}=(A x-y)^{T}(A x-y)
$$

To reduce the mean square error to a minimum, differentiating eqn. $\mathbf{4}$ partially with respect to $\mathbf{x}$ and equating to zero, we get

$$
A^{T} A x=A^{T} y
$$

Eqn. 5 is the well known 'normal equation'.

$$
\text { If } \boldsymbol{A}^{T} \boldsymbol{A} \text { is nonsingular, then }
$$

$$
\begin{aligned}
& \boldsymbol{x}=\left(A^{T} A\right)^{-1} A^{T} y \\
& \boldsymbol{x}=B \boldsymbol{y}
\end{aligned}
$$

where $\boldsymbol{B}=\left(\boldsymbol{A}^{T} \boldsymbol{A}\right)^{-1} \boldsymbol{A}^{T}$ is called as the pseudoinverse of the given matrix $A$.

Since the column of $\boldsymbol{A}^{T} \boldsymbol{A}$ matrix is linearly independent, a unique solution exists. A measure of the roundoff solution, close to the required solution, is given by the condition of the matrix $\boldsymbol{A}^{T} \boldsymbol{A}$. This matrix is said to be illconditioned if the determinant is very much less than its element, i.e. $\operatorname{det}\left(\boldsymbol{A}^{T} \boldsymbol{A}\right) \ll a_{i j}$, where $a_{i j}$ is an element of $\boldsymbol{A}^{T} \boldsymbol{A}$. Also $\boldsymbol{K}\left(\boldsymbol{A}^{T} \boldsymbol{A}\right)=\lambda_{\text {max }} / \lambda_{\min }$ should be small, where $K$ is the condition of the matrix, $I, \%$ and $\lambda_{\min }$ are the maximum and minimum eigen values of the matrix $\boldsymbol{A}^{T} \boldsymbol{A}$. The condition of the matrix is an important index when the signal is nonuniformly sampled.

\section{Digital computer analysis}

A digital computer program has been developed to calculate the harmonic component. The flow diagram is shown in Fig. 1. Here matrix $\boldsymbol{A}$ contains the coefficients of the trigonometric series of sine and cosine terms, hence it can be calculated in advance if the sampling period is known. Further, $\boldsymbol{A}^{T} \boldsymbol{A}$ is reduced to a $2 \times 2$ diagonal matrix (if uniform samples are considered) to calculate the required rth harmonic sine and cosine component (coefficient of the trigonometric series) and the multiplication needed now is between $A_{2 \times p}^{T}$ and $y_{p \times 1}$ matrices to evaluate $x_{2 \times 1}$. Therefore, only two components (sine and cosine) are tracked at a time, independently by the present method. It is not necessary to assume the nontracked harmonics to be zero. No complex multiplication is required since the elements of the matrix are in the real field. Calculations are done using an IBM PC compatible with following system configuration:

Processor type: 80386

Numeric coprocessor: 80387

Basic memory size: 640 Kbyte

Extend memory size: 7168 Kbyte

Hard drive: C: 171 Mbyte

Clock/calendar: CMOS clock/calendar present

Clock speed: $25 \mathrm{MHz}$

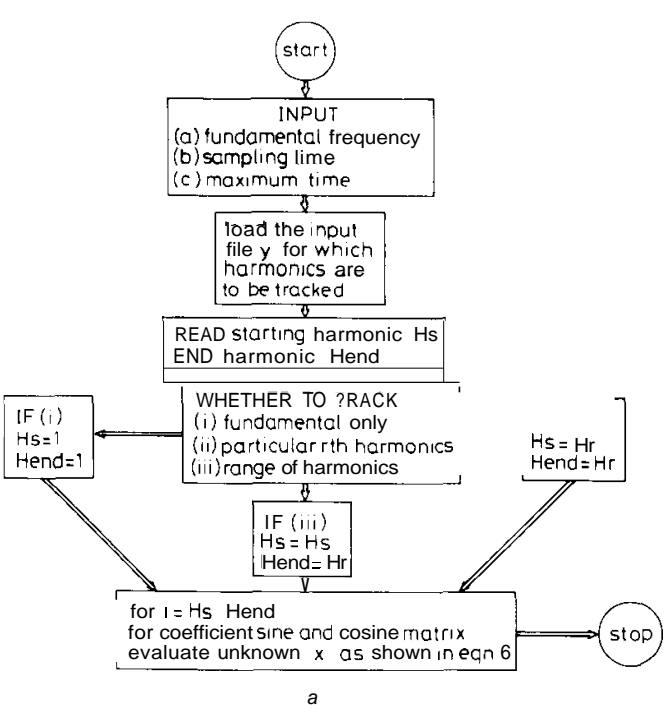

Fig. 1 Flow diagramjor calculating the harmonic component

\section{Results and discussion}

\subsection{Hypothetical signal analysis}

Many known signals were analysed to selectively track the harmonic components and the proposed SHT works well with these synthetic signals for both uniform and nonuniform sampling intervals. In a practical AC-DC system, the fundamental signal is quite often superimposed by partial disturbance during opening and closing of circuit breakers or disconnector switches. As shown in Fig. $2 a$, such a situation is synthesised by superimposing a piecewise noise over the fundamental $(50 \mathrm{~Hz})$, sampled at $10 \mathrm{kHz}$. The fundamental is successfully tracked by SHT along with the damped noise (Fig. 2b).

With the sinusoidal signal and uniformly distributed random noise, sampled at different intervals the $50 \mathrm{~Hz}$ fundamental is recovered whose magnitude variation is shown in Fig. 3. It is clear that, as the sampling rate is increased, the accuracy also increases even in the presence of noise. This is due to the Shanon sampling theorem. The optimum choice of sampling interval depends on the error that can be allowed and the number of harmonics which are significant from the study point of view.

Fig. 4 shows error analysis of FFT and SHT at various sampling intervals to track the harmonic components. Series 1 and 3 represent the 3rd and 5th harmonic component as calculated by FFT and series 2 and 4 correspond to that tracked by SHT. It can be observed 
that at the boundary value of the sampling rate $(0.5 \mathrm{kHz})$. which corresponds to the highest frequency component to be tracked (in this case 5th harmonic), the method of SHT gives the required harmonic components with an error of $4.5 \%$ for the 5 th and $7.5 \%$ for the $3 \mathrm{rd}$, whereas
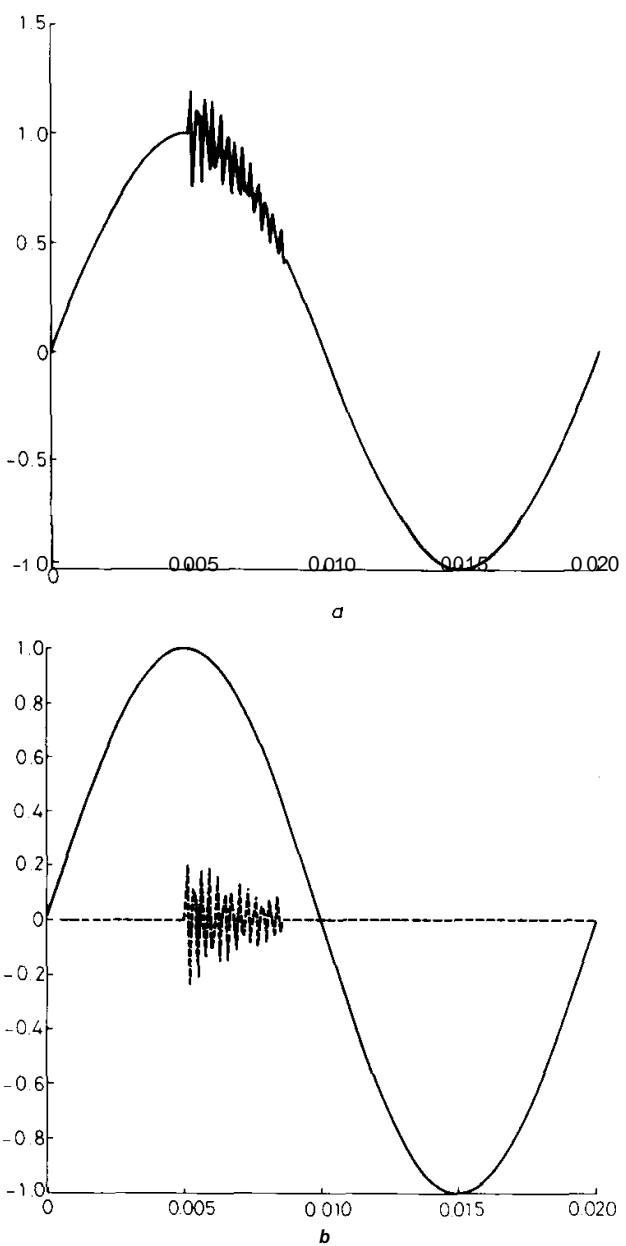

Fig. 2 Superimposing a piecewise noise over the fundamental $(50 \mathrm{~Hz}$ fundamenta!

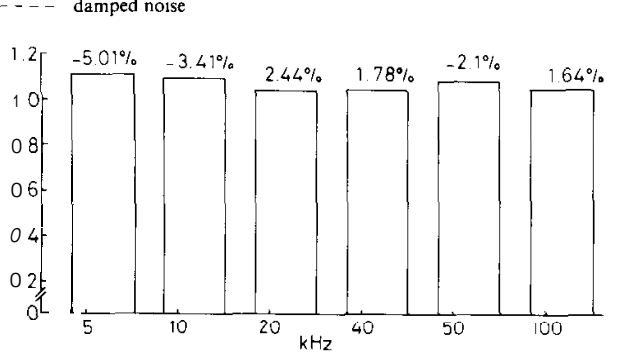

Fig. 3 Magnitude of the recovered $50 \mathrm{~Hz}$ fundamental sampled ar dif. ferent intervals

$y=\sin \omega t+\operatorname{rand} t$

the same components as found by FFT has an error of $35.7 \%$ and $20 \%$, respectively (the Hanning window is used for the FFT). As can be seen, when the sampling rate is increased, the FFT performs better whereas SHT gives actual harmonic magnitudes of 0.5 and 0.25 (5th and 3rd) with zero error after $0.5 \mathrm{kHz}$ sampling rate.

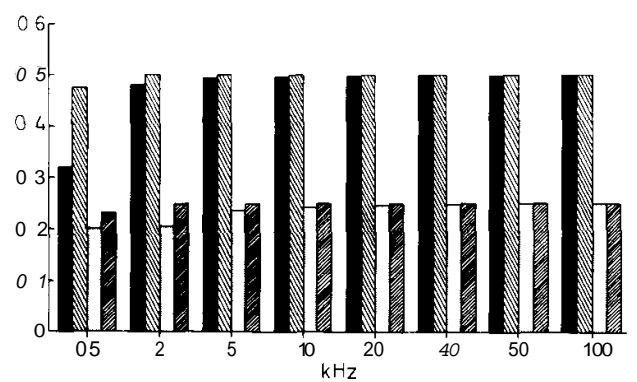

Fig. 4 Error analysis of FFT and SHT at various sampling interval - Series I (FFT)

Series 2 (SHT)

$\square$ Series 3 (FFT)

Series 4 (SHT)

$y=\sin \omega t+0.5 \sin 3 o t+0.25 \sin 5 \omega t$

\subsection{Integrated $A C / D C$ system studies}

A digital computer simulation of the MTDC (multiterminal direct current) system, whose schematic diagram is shown in Fig. 5, is carried out. It comprises

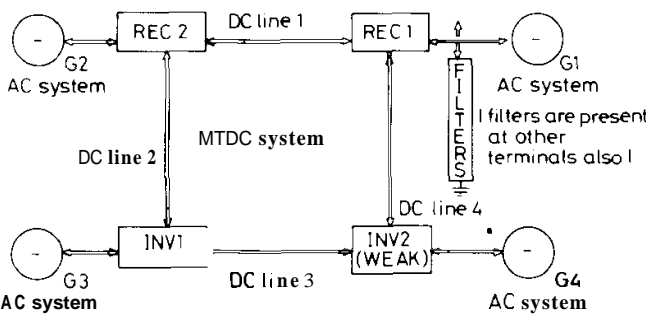

Fig. 5 Schematic diagramfor the digital simulation of the MTDC

four AC systems with two rectifiers and two inverter stations, one of which is connected to a weak AC system [11]. This system is analysed under a single line to ground (SLG) fault on the AC side of inverter 2 and the fault voltage is depicted in Fig. $6 a$ along with the selected fundamental using SHT. On the DC side of inverter 2 , lower order harmonics are tracked (Fig. 6b). This shows that the magnitudes of lower order harmonics give (1-4) important indices for recognising the voltage pattern under fault conditions

The synthesised DC voltage using inverse SHT is shown in Fig. 6c under SLG fault condition at inverter 2 taking 0 to 99 harmonics. Hence the proposed SHT is useful in tracking the required low frequency harmonic components during fault conditions from high frequency ripples in the $\mathbf{D C}$ voltage.

\subsection{Comparative analysis}

A comparative study is carried out with the FFT (fast Fourier transform) analysis for a simple sinusoidal signal of frequency $50 \mathrm{~Hz}$, consisting of only the fundamental, sampled at $10 \mathrm{kHz}$. The normalised components are compared as shown in Fig. 7. It is clear that apart from the fundamental, FFT has higher $\left(10^{-04}\right)$ magnitudes for other harmonics (strictly zero) compared to the present method (SHT) whose normalised values for harmonics other than fundamental is below $10^{-16}$ which can be 
considered as 'machine zero'. It was then seen that, even if the sampling period was increased to $100 \mathrm{kHz}$, the result did not improve with FFT compared to SHT.
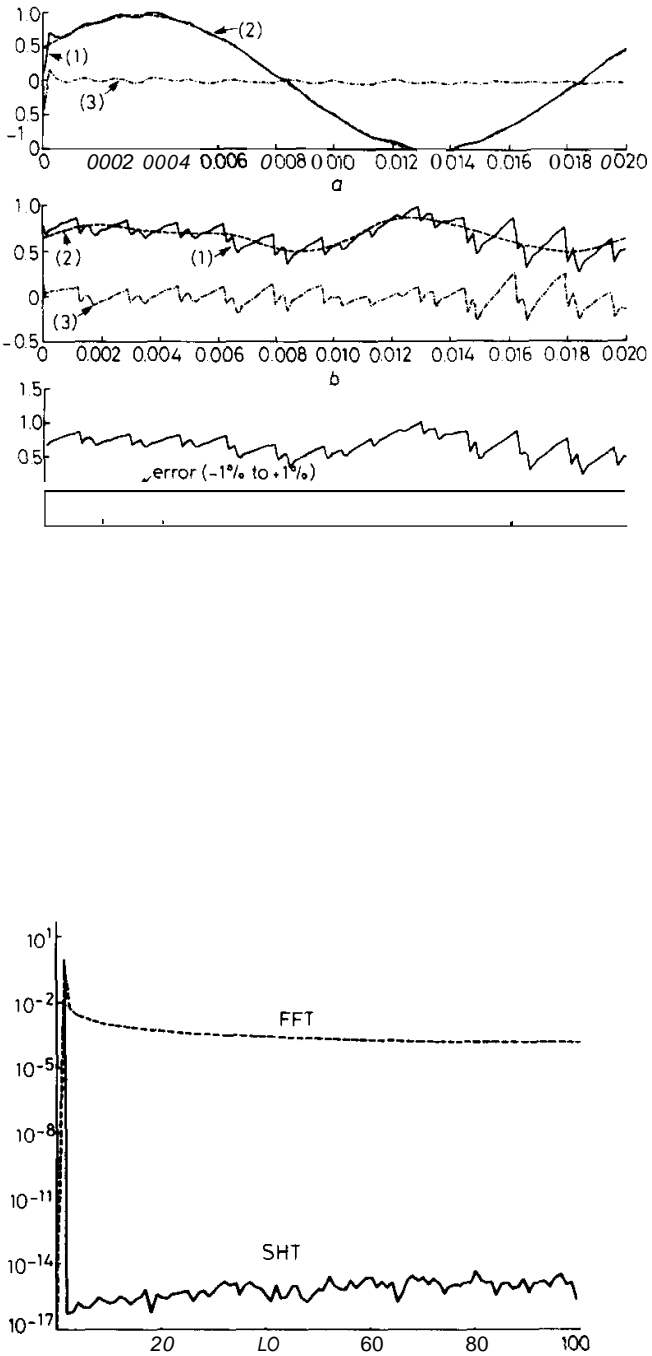

Fig. 7 Comparison of FFT and SHT for a simple sinusoidal signal $(50 \mathrm{~Hz})$ sampled at $10 \mathrm{kHz}$

Comparisonis with normalised components

It is difficult to compare the computation time taken by the present method and the other methods such as FFT because in the present method each harmonic sine and cosine component is calculated selectively at a time, whereas FFT calculates all the harmonic components in the Nyquist range. However, the elapsed time for tracking the fundamental at different sampling rates by the present method is depicted in Fig. 8. For sampling rates from 0.005 to $0.025 \mathrm{MHz}$ the time elapsed is almost constant $(0.05$ to 0.06 s. At $0.1 \mathrm{MHz}$ it rises to $\mathbf{0 . 2 8} \mathrm{s}$. As a comparison, the elapsed time to calculate the harmonic components at a sampling period of $10 \mathrm{kHz}$ for a $50 \mathrm{~Hz}$ fundamental signal over one period using the Hanning window by FFT is $2.2 \mathrm{~s}$ but it yields the harmonic spectrum from 1 to 100 .
The proposed SHT method is well-suited to the neural architectures discussed in recent literature $[12,13]$. Using neural principles, in conjunction with the present method it is possible to estimate the harmonic components in real time which gives unique solution (global minimum). The authors have obtained excellent convergence in the range

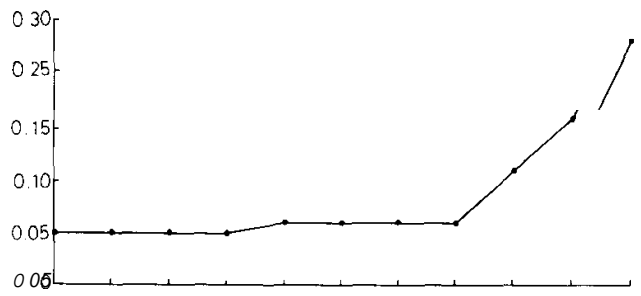

000500100011001250014300166002000250033005010 $\mathrm{MH}-\mathrm{z}$

Fig. 8 Elapsed time for tracking the fundamental at different sampling rates

of few microseconds through the computational analysis [14]. The method of SHT does not use any windowing function, crosscorrelation etc., for reconstructing the wave form. When the signal is highly aperiodic (discontinuous), due to Gibb's phenomena, only the starting value (after synthesis) would be high in the present method. Using the proper arithmatic sequence [9], the effect due to Gibbs' phenomena can be reduced.

\section{Conclusions}

A simple method of selectively tracking the harmonics is developed. This type of analysis is helpful in analysing the harmonic pollution effect; especially in an integrated AC-DC system which incorporates filters and SVCs and in digital relaying. The utility of the SHT method can be summarised as follows:

(i) It can track the required harmonic components from the transient signals over the fundamental range.

(ii) It can analyse the signals with piecewise superimposed low frequency noise as it is the case with the converter station in MTDC-AC system.

(iii) It may be used as an estimator of the future values of the function using a finite Taylor's series because all the derivatives for a function expressed in a trigonometric series consisting of sine and cosine terms exist.

The concept developed may be used in conjunction with the neural network to estimate the harmonic components which give a global minimum solution in real time.

\section{References}

1 ORAN BRIGHAM, E.: 'The fast Fourier transformer and its applications' (Prentice-Hall, Reading, MA, 1988)

2 KARL, J.H.: 'An introduction to digital signal processing' (Academic Press, Harcourt Brace Jovanovich, San Diego, CA, 1989) 3 HEYDT, G.T.: 'Harmonics in power systems'. Tutorial notes provided at the 6th National Power System Conference, Bombay, India, 4th-7th June 1990, Section 7.0

4 DASH, P.K., and SHARAF, A.M.: 'A Kalman filtering approach for estimation of power system harmonics'. Proceedings of the 3rd international conference on Harmonics in Power Systems, Nashville, IN, 28th Sept.-1st Oct. 1988,pp. 34-40

5 BEIDES, H.M., and HEYDT, G.T.: 'Dynamic state estimation of 5 BEIDES, H.M., and HEYDT, G.T.: 'Dynamic state estimation of power system harmonics using Kalman filter methodo
Trans. Power Delivery, Oct. 1991, 6, (4), pp. 1663-1670

6 HEYDT, G.T., KISH, D.J., HOLCOMB, F., and HILL, Y.: 'A methodology for assessment of harmonic impact and compliance with standards for distribution systems', IEEE Trans. Power Delivery, Oct. 1991, 6, (4), pp. 1748-1754 
7 MORI, $\mathrm{H}$, TSUZUKI, S, SAKURAI, T, KOJIMA, $\mathrm{Y}$, and SATOH, N.: 'Identification of harmonic current characteristics in power systems'. Proceedings of the 3rd international conference on Harmonics in Power Systems, Nashville, IN, 28th Sept.-1st Oct. 1988, pp. 78-84

8 HARTANA, R.K., and RICHARDS, G.H.: 'Harmonic source monitoring and identification using neural networks', IEEE Trans. Power Systems, Nov. 1990, 5, (4), pp. 1098-1104

9 GUILLEMIN, E.A.: 'The mathematics of circuit analysis' (Oxford and IBH Publishing, 1962), Chap. 7

10 PHADKE, G., and THORP, J.S.: 'Computer relaying for power systems' (Research Studies Press, 1988), Chap. 3

11 MANOHAR, P., and CHANDRASEKHARAIAH, H.S.: 'Application of $\mathrm{Zno}$ varistor protection to capacitors of artificially commut* ated inverters in MTDC system', IEEE Trans. Power Systems, 1991, 6. (2). pp. $356-363$

12 CICHOCKI, A., and UNBEHAUEN, R.: 'Neural networks for solving systems of linear equations and related problems', IEEE Trans. Circuits \& Syst. 1: Fundamental Theory and Applications, Feb. 1992, CAS-39, (2), pp. 124-137

13 OSOWSKI, S.: 'Neural network for estimation of harmonic components in a power system', IEE Proc. C, March 1992, 139, (2), pp. 129-135

14 NARENDRA, K.G., and CHANDRASEKHARAIAH, H.S.: 'Tuned neural architecture for detecting harmonics in a MTDC-AC system'. Paper submitted for PICA '93, Phoenix AZ, 1993, 4th-7th May 